\title{
CHOLINERGIC MODULATION OF MEMORY
}

\author{
RALUCA ELENA MOISESCU ${ }^{1 \#}$, ISABEL CRISTINA VIORICA GHIȚA ${ }^{1 * *}$, ADELINA SORESCU $^{1 \#,}$ \\ OANA ANDREIA COMAN ${ }^{1 \#}$, LAURENȚIU COMAN $^{2 \#}$, ION FULGA $^{1 \#}$
}

\author{
"Carol Davila" University of Medicine and Pharmacy, Faculty of Medicine \\ ${ }^{I}$ Department of Pharmacology and Pharmacotherapy \\ ${ }^{2}$ Department of Physiology
}

*correspondingauthor: isabelghita@yahoo.co.uk

\#All authors have equal contribution to this paper.

\begin{abstract}
Memory development is defined as the ability to encode, store and access new information. Certain neurotransmitters, but most importantly the cholinergic transmission via nicotinic receptors, have been linked not only to memory formation but to attention and other cognitive processes as well. In order to analyse previous literature on this subject a PubMed search was performed and it concluded 63 articles. We determined the overall efficacy and the prevalence of specific tests used. The implication of the cholinergic transmission via nicotinic receptors in memory has been well established over the years. Since the evolvement of technology, the nicotinic receptors have been linked not only to normal cognitive functions, but to the pathophysiology of certain neurodegenerative diseases as well. Due to their important roles in cognition, further studies need to be performed in order to determine their potential therapeutic effects.
\end{abstract}

\section{Rezumat}

Formarea memoriei este definită ca fiind capacitatea de a decoda, stoca și accesa noi informații. Anumiți neurotransmițători, dar în special sistemul colinergic prin intermediul receptorilor nicotinici, au fost corelați nu doar cu memoria, dar și cu atenția și alte procese cognitive. Pentru a analiza publicațiile existente pe această tematică, am utilizat motorul de căutare PubMed și am selectat un număr de 63 de articole. Am determinat relevanța și prevalența testelor specifice utilizate în respectivele studii. De-a lungul timpului, în literatura de specialitate s-a arătat că sistemul colinergic prin intermediul receptorilor nicotinici deține un rol important în controlul funcțiilor mnezice prin intermediul diferitelor tipuri de receptori nicotinici. Datorită dezvoltării tehnologiei, s-a dovedit că acești receptori nu sunt implicați doar în funcțiile fiziologice ale cogniției, ci și în fiziopatologia bolilor neurodegenerative. Ținând cont de importanța acestor receptori în procesul cognitiv, studiile ulterioare vor trebui să determine potențialul terapeutic al diverselor substanțe ce acționează asupra acestor receptori.

Keywords: nicotine, memory, cholinergic modulation

\section{Introduction}

Memory is a complex process that involves both storing and retrieving specific pieces of information. Even though these two actions are interconnected, being able to retain certain facts does not necessarily involve the ability to access them. This can usually be explained by the vulnerability of recently acquired memories [12]. Memory has been defined as the ability to retain information and recollect previous experiences [12]. When receiving new data, three main transformations are performed - storage, consolidation and retrieval. First of all data are encoded, a process that consists of receiving and processing the information (Figure 1). Second of all, it has been established that new memories are prone to disruption. That is why they need to be stored for future use. This process is called consolidation. Last but not least, by strengthening the pieces of information, they can be recalled and expressed as behaviour [10] (recall phase). Apart from this, memories are subjected to other processes over the time. For example, during the retrieval of information, a labilization process can occur, thus making the memories sensitive again [45]. What Blake et al. [12] observed was that a continuous reconsolidation is necessary for memories to become permanent.

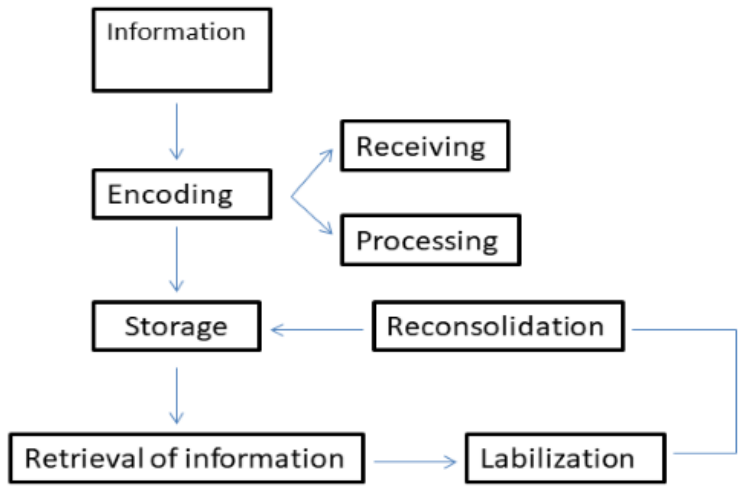

Figure 1.

Information processing during memory development 
FARMACIA, 2018, Vol. 66, 6

Each and every one of this process is mediated via neurotransmitter systems. Even though the most reported modulatory system is the cholinergic one, other transmitters have been associated with memory development, for example, the dopaminergic, serotoninergic and glutamate system.

Thus, in order to become permanent, information must be subjected to a number of cognitive processes, all mediated by chemical molecules. Certain neurotransmitters have been linked not only to memory formation but to attention and other cognitive functions as well, but by far the cholinergic transmission via nicotinic receptors is the most extensively studied one. Generally speaking, whether implying the administration of nicotinic agonists or observing the effects of acute or chronic smoking, a large number of studies were performed in order to demonstrate the implication of nicotinic receptors in memory. Most of them concluded that nicotine and other agonists improve memory acquisition $[6,43,49,56]$. Faster information processing [59], increased attention [57], improved spatial and working memory [1] are only some of the effects of nicotinic stimulation previously reported in the literature.

The nicotinic receptors are one of the two main types of cholinergic receptors. Two major receptor subtypes can be identified within the brain: the alpha $7(\alpha 7)$ and alpha-4 beta-2 ( $\alpha 4 \beta 2)$ nicotinic receptors. In general, nicotinic cholinergic receptors (nAChRs) are pentameric complexes of $\alpha$ - and $\beta$-subunits that form a ligandgated ion channel (Figure 2). The $\alpha 7 \mathrm{nAChR}$ is the most prevailing member of the neurotransmitter-gated ion channel superfamily. The ones localised in the central nervous system are homopentamers composed of five $\alpha 7$ subunits. However, assembly of $\alpha 7$ subunits to a functional homopentameric receptor requires the help of Ric-3 protein and/or post-translational processing [26]. Situated especially in the hippocampus, these receptors mediate an excitatory synaptic transmission [3]. However, the major heteromeric $\mathrm{nAChR}$ found in the nervous system is $\alpha 4 \beta 2$, which most likely contains two $\alpha 4$ subunits and three $\beta 2$ subunits. The functions of the $\alpha 4 \beta 2$ subtype have not yet been fully identified. However, these receptors have been correlated with the pathophysiology of Alzheimer's disease [47] and memory impairment determined either by nicotine withdrawal [63] or long-term stress [4]. Even though both receptor subtypes seem to have the same stimulating effect on memory, Kroker et al. [30] concluded that $\alpha 7$ receptor agonists are more efficient in memory improvement than $\alpha 4 \beta 2$ agonists. Apart from the addiction induced by nicotine, this chemical compound has other pharmacological effects [31]. The implication of the cholinergic system (especially the nicotinic receptors situated in the brain) in memory formation has been well established over the years in a large number of studies [49]. Aside from this, both nicotine and other agonists help improve motor performances [61], enhance attention [57] and alleviate stress and any cognitive deficits determined by it $[4,33]$.

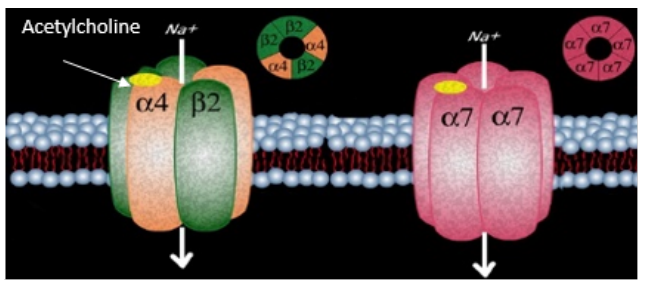

Figure 2.

Comparison between the two nicotinic receptors' structure

(Modified after www.jyi.org/wp-content/uploads//img04.jpg)

Numerous studies observed a connection between different neurotransmitter systems. Within the brain, different nicotinic receptors form complexes with other receptors, such as the dopaminergic and serotoninergic receptors and act sinergically to improve memory. The link between nicotinic and dopaminergic receptors was the most reported one $[1,13,28,36]$. It was observed that other established connections were with serotoninergic receptors [58] and glutamate receptors $[30,54]$, especially NMDA receptors $[14,19,27,55]$.

All these effects alongside the implication of nicotinic receptors in different conditions infer therapeutic possibilities. In neurodegenerative diseases such as Alzheimer's disease, Parkinson's disease, a decrease in the number of nicotinic receptors has been seen [11]. Apart from this, the stimulation of $\alpha 7$ receptors had impressive results, improving cognitive function. The treatment possibility has been explored with the help of clinical trials. For example, a trial with a $\alpha 7$ agonist has been conducted on subjects with schizophrenia and there was a statistically significant effect on working memory [39].

The effects produced by the complexes between nicotinic receptors and dopaminergic, serotoninergic and NMDA receptors need to be further studied due to the probable clinical uses of this neurotransmitter (nicotine).

This review approaches both physiological and pathological aspects of the importance of the cholinergic transmission via nicotinic receptors in memory development.

\section{Materials and Methods}

In order to analyse previous literature published on this theme, a PubMed search of the articles issued between 1981 and 2016, was performed. We used three key-words combinations. First of all we used "nicotine", "receptors" and "memory", to find general pieces of information regarding the implication of nicotinic receptors in the development of memory. We additionally applied a filter for " $\alpha 7$ " and " $\alpha 4 \beta 2$ " nicotinic receptor subtypes, for retrieving such details. 
Moreover, in order to avoid duplicating the data, we eliminated any meta-analyses. Our PubMed search method concluded with a number of 63 articles. We determined the overall efficacy and the prevalence of specific tests used. Apart from this, we categorised the studies depending on the used subjects (animals or humans).

\section{Results and Discussion}

A total of 63 articles have been retrieved after our PubMed search. Amongst these, there were 31 articles referring to the receptor subtypes, namely $\alpha 7$ and $\alpha 4 \beta 2$. The rest of them contained general information about the involvement of nicotinic receptors in cognitive processes. The selected articles were published during a wide time range, from 1981 until 2016, which enabled us to observe the knowledge acquired over the years.

Only three of these articles were literature reviews, the rest of them being original studies. We tried to categorise these, depending on: the subjects used, the tests applied and their results.

First of all we found impressive the fact that only one article published by Mundi et al. [43] (representing $2 \%$ ) reported a negative response following nicotine administration (Figure 3). To be more precise, this article concluded that high-doses of nicotine reduced memory development.

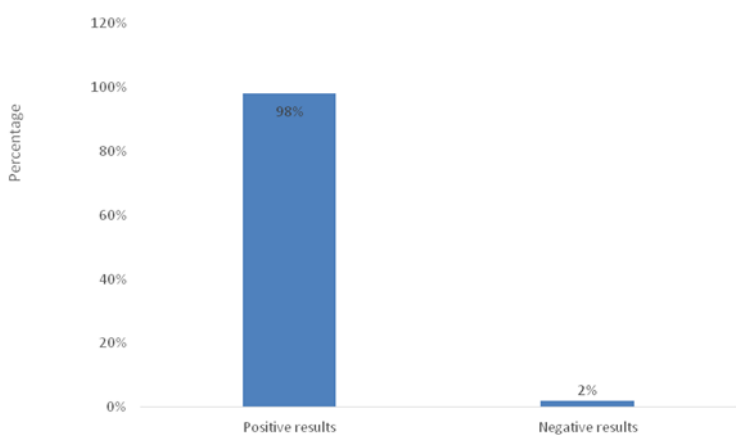

Figure 3.

The number of positive and negative results of the studies on nicotine improvement of memory

These studies were performed on different subjects. The most prevailing ones were male Sprague-Dawley rats $(61 \%)$, followed by different species of mice (with a frequency of $21 \%$ ) and only 3 clinical trials on humans (5\%) (Figure 4).
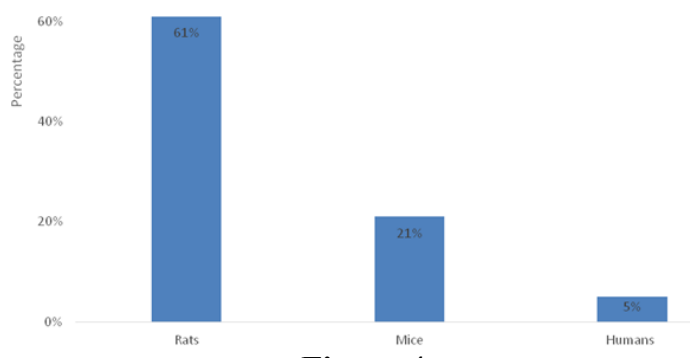

Figure 4.

The subjects used in the analysed studies

The study designs differed greatly. The most common test used to determine the effects of nicotinic receptor agonists was the radial arm maze (17 out of 63 studies). Another 7 studies performed a Morris water maze. Apart from these, multiple T-mazes (5 studies), inhibitory avoidance behaviour and passive avoidance response (each used 4 times), novel object recognition tasks and five-choice serial reaction time tasks (both utilised 3 times) were used. Other tests used, but on a small number of occasions, were the Y-maze test, the Lashley III maze test, Barnes maze test, object location tasks, open field tests, attentional set-shifting tasks, active avoidance tasks and odour span tasks (Figure 5). Each of these tested different cognitive processes (Table I and Table II). The oldest study selected that reported the connection between the central cholinergic systems and learning and memory was published in 1985. In this article, Haroutunian et al. [23] concluded that administration of cholinergic agonists (such as arecoline) potentiated the retention phase of memory in rats. However, the agonist dosage had been reported to influence the effect on cognition [20]. These results have been duplicated by Haroutunian et al. [23],

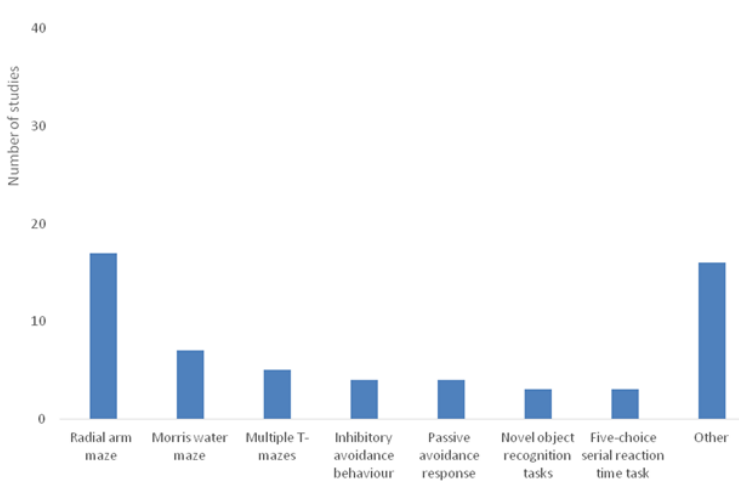

Figure 5.

The main tests used in animals to determine the effects of nicotine on memory 
Studies using animal subjects (sorted by the tests used)

\begin{tabular}{|c|c|c|c|c|c|}
\hline Author, Year & Subjects & Tests utilised & $\begin{array}{l}\text { Evaluated } \\
\text { Parameter }\end{array}$ & Chemical compounds & Comments \\
\hline Perio, 1989 & Wistar rats & - & $\begin{array}{l}\text { Retroactive } \\
\text { facilitation }\end{array}$ & $\begin{array}{c}\text { Physostigmine salicylate, Arecoline } \\
\text { hydrobromide, RS86, Nicotine } \\
\text { sulfate, Strychnine sulfate, } \\
\text { Scopolamine hydrobromide, } \\
\text { Scopolamine methylbromide } \\
\end{array}$ & $\begin{array}{l}\text { Cholinomimetic drugs } \\
\text { facilitate social memory }\end{array}$ \\
\hline Kroker, 2011 & Wistar rats & - & $\begin{array}{l}\text { Hippocampal } \\
\text { long-term } \\
\text { potentiation }\end{array}$ & TC-1827 and SSR180711 & $\begin{array}{c}\text { SSR180711 showed } \\
\text { enhancing effects on both } \\
\text { early and late LTP }\end{array}$ \\
\hline Mundy, 1988 & $\begin{array}{l}\text { Sprague } \\
\text { Dawley } \\
\text { rats }\end{array}$ & $\begin{array}{l}8 \text {-arm radial } \\
\text { maze }\end{array}$ & $\begin{array}{l}\text { Working } \\
\text { memory, } \\
\text { Reference } \\
\text { memory }\end{array}$ & Nicotine, Scopolamine & $\begin{array}{l}\text { Nicotine impairs memory } \\
\text { acquisition }\end{array}$ \\
\hline Baraldi, 2013 & Mice & Barnes maze & Spatial memory & - & $\begin{array}{l}\text { Nicotinic system improves } \\
\text { memory }\end{array}$ \\
\hline Liu, 2004 & Wistar rats & $\begin{array}{l}\text { Delayed non- } \\
\text { matching-to- } \\
\text { position task }\end{array}$ & Spatial memory & Nicotine, Atropine & $\begin{array}{c}\text { Muscarinic receptors had a } \\
\text { memory-enhancing effect if } \\
\text { the nicotinic ones were } \\
\text { desensitised }\end{array}$ \\
\hline $\begin{array}{l}\text { Stolerman, } \\
2000\end{array}$ & $\begin{array}{l}\text { Hooded } \\
\text { rats }\end{array}$ & $\begin{array}{l}\text { Five-choice } \\
\text { serial reaction } \\
\text { time task }\end{array}$ & $\begin{array}{l}\text { Memory, } \\
\text { attention }\end{array}$ & $\begin{array}{c}\text { Nicotine bitartrate, } \\
\text { Mecamylamine, Scopolamine }\end{array}$ & $\begin{array}{c}\text { Mecamylamine produced a } \\
\text { modest deficit in reaction } \\
\text { time }\end{array}$ \\
\hline Yildirim, 2015 & Mice & $\begin{array}{l}\text { Five-choice } \\
\text { serial reaction } \\
\text { time task } \\
\end{array}$ & $\begin{array}{c}\text { Fear } \\
\text { conditioning }\end{array}$ & $\begin{array}{c}\text { ABT-107, ABT-1089, disolved in } \\
\text { saline }\end{array}$ & $\begin{array}{c}\text { Only ABT-1089 reverses } \\
\text { nicotine withdrawal-induced } \\
\text { deficits }\end{array}$ \\
\hline Brioni, 1993 & Mice & $\begin{array}{l}\text { Inhibitory } \\
\text { avoidance } \\
\text { response }\end{array}$ & $\begin{array}{l}\text { Memory } \\
\text { retention }\end{array}$ & Nicotine, Cytisine, Lobeline & $\begin{array}{l}\text { Nicotine agonists facilitate } \\
\text { memory acquisition }\end{array}$ \\
\hline Ballesta, 2012 & Wistar rats & $\begin{array}{c}\text { Inhibitory } \\
\text { avoidance task }\end{array}$ & $\begin{array}{l}\text { Memory } \\
\text { retention }\end{array}$ & - & $\begin{array}{c}\text { Reduction } \alpha 4 \beta 2 \mathrm{nAChRs} \\
\text { determines cognitive } \\
\text { dysfunction }\end{array}$ \\
\hline Blake, 2012 & Mice & $\begin{array}{c}\text { Inhibitory } \\
\text { avoidance task }\end{array}$ & $\begin{array}{l}\text { Memory } \\
\text { retention }\end{array}$ & $\begin{array}{c}\text { Scopolamine hydrochloride, } \\
\text { scopolamine methyl-bromide and } \\
\text { choline bitartrate }\end{array}$ & $\begin{array}{c}\text { Choline reverses } \\
\text { scopolamine's effects }\end{array}$ \\
\hline Blake, 2013 & Mice & \begin{tabular}{|c|} 
Inhibitory \\
avoidance task
\end{tabular} & $\begin{array}{l}\text { Memory } \\
\text { retention }\end{array}$ & Choline & $\begin{array}{c}\text { Nicotinic system improves } \\
\text { memory }\end{array}$ \\
\hline Morris, 2010 & $\begin{array}{l}\text { Fischer- } \\
344 \text { rats }\end{array}$ & \begin{tabular}{|c|} 
Inhibitory \\
avoidance task
\end{tabular} & $\begin{array}{l}\text { Memory } \\
\text { retention }\end{array}$ & $\begin{array}{l}\text { Methyllycaconitine citrate salt } \\
\text { hydrate and Dihydro-b- } \\
\text { erythroidine hydrobromide }\end{array}$ & \\
\hline Guo, 2011 & Wistar rats & $\begin{array}{l}\text { Morris water } \\
\text { maze }\end{array}$ & $\begin{array}{c}\text { Spatial learning } \\
\text { and memory }\end{array}$ & Scutellarin & $\begin{array}{c}\text { Scutellarin has protective } \\
\text { effects on rats with memory } \\
\text { deficits } \\
\end{array}$ \\
\hline $\begin{array}{l}\text { Muthuraju, } \\
2011\end{array}$ & $\begin{array}{l}\text { Sprague } \\
\text { Dawley } \\
\text { rats }\end{array}$ & $\begin{array}{l}\text { Morris water } \\
\text { maze }\end{array}$ & Spatial memory & $\begin{array}{c}\text { Acetylcholine iodide, } \\
\text { Physostigmine, Galantamine }\end{array}$ & $\begin{array}{l}\text { Nicotinic system improves } \\
\text { memory }\end{array}$ \\
\hline \begin{tabular}{|c|} 
Sadigh- \\
Eteghad, 2015
\end{tabular} & Mice & $\begin{array}{l}\text { Morris water } \\
\text { maze }\end{array}$ & $\begin{array}{l}\text { Working and } \\
\text { reference } \\
\text { memory }\end{array}$ & PHA-543613 & $\begin{array}{c}\text { a7 nAChR activation has } \\
\text { neuroprotective effects in } \\
\text { Alzheimer's disease }\end{array}$ \\
\hline Socci, 1995 & $\begin{array}{l}\text { Sprague } \\
\text { Dawley } \\
\text { rats }\end{array}$ & $\begin{array}{l}\text { Morris water } \\
\text { maze }\end{array}$ & $\begin{array}{l}\text { Memory } \\
\text { retention, } \\
\text { attention }\end{array}$ & Nicotine & $\begin{array}{l}\text { The results suggest that } \\
\text { nicotine can treat memory } \\
\text { deficits in Alzheimer's disease }\end{array}$ \\
\hline Zhou, 2004 & Rats & $\begin{array}{l}\text { Morris water } \\
\text { maze }\end{array}$ & Spatial memory & Nicotine & $\begin{array}{c}\text { Nicotinic agonist treatments } \\
\text { can ameliorate learning and } \\
\text { memory impairments } \\
\end{array}$ \\
\hline Falsafi, 2012 & Mice & $\begin{array}{l}\text { Multiple T- } \\
\text { maze }\end{array}$ & $\begin{array}{l}\text { Spatial learning } \\
\text { and memory }\end{array}$ & Scopolamine & $\begin{array}{l}\text { Muscarinic receptors and } \\
\text { NMDA are involved in } \\
\text { memory formation }\end{array}$ \\
\hline \begin{tabular}{|c|} 
Subramaniyan, \\
2014
\end{tabular} & Mice & $\begin{array}{l}\text { Multiple T- } \\
\text { maze }\end{array}$ & $\begin{array}{l}\text { Spatial learning } \\
\text { and memory }\end{array}$ & - & $\begin{array}{c}\text { There was a correlation } \\
\text { between histaminergic and } \\
\text { nicotinic receptors }\end{array}$ \\
\hline
\end{tabular}


FARMACIA, 2018, Vol. 66, 6

\begin{tabular}{|c|c|c|c|c|c|}
\hline Author, Year & Subjects & Tests utilised & $\begin{array}{l}\text { Evaluated } \\
\text { Parameter }\end{array}$ & Chemical compounds & Comments \\
\hline $\begin{array}{c}\text { Vandesquille, } \\
2013\end{array}$ & Mice & $\begin{array}{l}\text { Multiple T- } \\
\text { maze }\end{array}$ & $\begin{array}{l}\text { Working } \\
\text { memory }\end{array}$ & S 38232 & $\begin{array}{c}\alpha 4 \beta 2 \text { agonists restores working } \\
\text { memory in aged mice }\end{array}$ \\
\hline $\begin{array}{c}\text { Lange- } \\
\text { Asschenfeldt, } \\
2016\end{array}$ & Mice & $\begin{array}{l}\text { Novel object } \\
\text { recognition }\end{array}$ & $\begin{array}{l}\text { Recognition } \\
\text { memory }\end{array}$ & Varenicline & $\begin{array}{c}\text { Nicotinic system improves } \\
\text { memory }\end{array}$ \\
\hline $\begin{array}{c}\text { Sadigh- } \\
\text { Eteghad, } 2015\end{array}$ & Mice & $\begin{array}{l}\text { Novel object } \\
\text { recognition }\end{array}$ & $\begin{array}{l}\text { Recognition } \\
\text { memory and } \\
\text { neurovascular } \\
\text { coupling }\end{array}$ & $\begin{array}{l}\text { PHA-543613, galantamine and } \\
\text { methyllycaconitine }\end{array}$ & $\begin{array}{l}\text { Nicotinic system improves } \\
\text { memory }\end{array}$ \\
\hline $\begin{array}{l}\text { Werkheiser, } \\
2011\end{array}$ & Mice & $\begin{array}{l}\text { Novel object } \\
\text { recognition }\end{array}$ & $\begin{array}{l}\text { Recognition } \\
\text { memory }\end{array}$ & AZD0328 & $\begin{array}{c}\text { Nicotinic system improves } \\
\text { memory }\end{array}$ \\
\hline $\begin{array}{l}\text { Karamihalev, } \\
2014\end{array}$ & Mice & $\begin{array}{c}\text { Object } \\
\text { location task }\end{array}$ & Spatial memory & $\begin{array}{l}\text { MK-801, donepezil and PHA } \\
568487\end{array}$ & $\begin{array}{l}\text { Nicotinic system improves } \\
\text { memory, alongside the } \\
\text { NMDA receptor }\end{array}$ \\
\hline Puma, 1999 & Wistar rats & $\begin{array}{l}\text { Object } \\
\text { recognition } \\
\text { task }\end{array}$ & $\begin{array}{l}\text { Working } \\
\text { memory }\end{array}$ & Nicotine & $\begin{array}{l}\text { Nicotine improves memory } \\
\text { retention }\end{array}$ \\
\hline $\begin{array}{l}\text { Rushforth, } \\
2010\end{array}$ & Rats & $\begin{array}{l}\text { Odour span } \\
\text { task }\end{array}$ & $\begin{array}{l}\text { Olfactory } \\
\text { working } \\
\text { memory }\end{array}$ & Metanicotine, Compound A & $\begin{array}{c}\text { Both receptor subtypes } \\
\text { enhance olfactory working } \\
\text { memory }\end{array}$ \\
\hline Eidi, 2003 & Wistar rats & $\begin{array}{c}\text { Passive } \\
\text { avoidance task }\end{array}$ & $\begin{array}{l}\text { Memory } \\
\text { retention }\end{array}$ & $\begin{array}{l}\text { Histamine dihydrochloride, } \\
\text { Pyrilamine maleate, Cimetidine, } \\
\text { Acetylcholine dihydrochloride, } \\
\text { Scopolamine, Nicotine }\end{array}$ & $\begin{array}{l}\text { Nicotinic system improves } \\
\text { memory }\end{array}$ \\
\hline Smith, 1996 & $\begin{array}{l}\text { Sprague } \\
\text { Dawley } \\
\text { rats }\end{array}$ & $\begin{array}{l}\text { Passive- } \\
\text { avoidance } \\
\text { response }\end{array}$ & $\begin{array}{l}\text { Memory } \\
\text { retention }\end{array}$ & $\begin{array}{c}\text { Arecoline, Nicotine, Pilocarpine, } \\
\text { Oxotremorine, E2020, Tacrine, } \\
\text { Mecamylamine, Atropine and } \\
\text { Scopolamine }\end{array}$ & $\begin{array}{l}\text { Nicotinic agonists improve } \\
\text { memory retention }\end{array}$ \\
\hline Welzl, 1988 & $\begin{array}{l}\text { Sprague } \\
\text { Dawley } \\
\text { rats }\end{array}$ & $\begin{array}{l}\text { Radial and } \\
\text { hexagonal } \\
\text { maze }\end{array}$ & $\begin{array}{c}\text { Locomotion and } \\
\text { exploratory } \\
\text { behaviour }\end{array}$ & Nicotine hydrogen & $\begin{array}{c}\text { nicotine treatment consistently } \\
\text { increased locomotion }\end{array}$ \\
\hline $\begin{array}{c}\text { Shanmugasun } \\
\text { daram,2015 }\end{array}$ & $\begin{array}{c}\text { Sprague } \\
\text { Dawley } \\
\text { rats }\end{array}$ & $\begin{array}{l}\text { Radial arm } \\
\text { maze }\end{array}$ & $\begin{array}{l}\text { Working } \\
\text { memory }\end{array}$ & - & $\begin{array}{c}\text { The nicotinic system forms } \\
\text { receptor complexes with } \\
\text { other systems }\end{array}$ \\
\hline Alzoubi, 2013 & Wistar rats & $\begin{array}{l}\text { Radial arm } \\
\text { water maze }\end{array}$ & $\begin{array}{l}\text { Learning and } \\
\text { memory } \\
\text { performance }\end{array}$ & $\begin{array}{l}\text { Methyllycaconitine, Dihydro-b- } \\
\text { erythroidine hydrobromide }\end{array}$ & $\begin{array}{c}\text { The a7-nAChRs are involved } \\
\text { in the neuroprotective effect } \\
\text { of nicotine }\end{array}$ \\
\hline Addy, 2002 & $\begin{array}{l}\text { Sprague } \\
\text { Dawley } \\
\text { rats }\end{array}$ & $\begin{array}{l}\text { Radial-Arm } \\
\text { Maze }\end{array}$ & $\begin{array}{l}\text { Working } \\
\text { memory } \\
\text { performance }\end{array}$ & $\begin{array}{l}\text { Haloperidol, Nicotine, } \\
\text { Risperidone, Clozapine }\end{array}$ & $\begin{array}{c}\text { Nicotine enhances memory } \\
\text { performance }\end{array}$ \\
\hline Addy, 2003 & $\begin{array}{l}\text { Sprague } \\
\text { Dawley } \\
\text { rats }\end{array}$ & $\begin{array}{l}\text { Radial-Arm } \\
\text { Maze }\end{array}$ & $\begin{array}{l}\text { Working } \\
\text { memory }\end{array}$ & $\begin{array}{l}\text { Methyllycaconitine, Dihydro-b- } \\
\text { erythroidine hydrobromide }\end{array}$ & $\begin{array}{l}\text { Nicotine improves working } \\
\text { memory }\end{array}$ \\
\hline Bettany, 2001 & $\begin{array}{l}\text { Sprague } \\
\text { Dawley } \\
\text { rats }\end{array}$ & $\begin{array}{l}\text { Radial-Arm } \\
\text { Maze }\end{array}$ & $\begin{array}{l}\text { Working } \\
\text { memory }\end{array}$ & $\begin{array}{c}\text { Nicotine, Mecamylamine, } \\
\text { Dihydro-b-erythroidine, } \\
\text { Methyllycaconitine }\end{array}$ & $\begin{array}{l}\text { Nicotinic system enhances } \\
\text { memory }\end{array}$ \\
\hline Levin, 1992 & $\begin{array}{l}\text { Sprague } \\
\text { Dawley } \\
\text { rats }\end{array}$ & $\begin{array}{l}\text { Radial-arm } \\
\text { maze }\end{array}$ & $\begin{array}{l}\text { Learning and } \\
\text { memory } \\
\text { performance }\end{array}$ & Nicotine & $\begin{array}{c}\text { The nicotinic system } \\
\text { improves memory formation }\end{array}$ \\
\hline Levin, 1995 & $\begin{array}{l}\text { Sprague } \\
\text { Dawley } \\
\text { rats }\end{array}$ & $\begin{array}{l}\text { Radial-arm } \\
\text { maze }\end{array}$ & $\begin{array}{l}\text { Working } \\
\text { memory } \\
\text { performance }\end{array}$ & Nicotine & $\begin{array}{l}\text { Acute administration } \\
\text { improved memory, while } \\
\text { chronic use reduced it }\end{array}$ \\
\hline Levin, 1996 & $\begin{array}{l}\text { Sprague } \\
\text { Dawley } \\
\text { rats }\end{array}$ & $\begin{array}{l}\text { Radial-arm } \\
\text { maze }\end{array}$ & $\begin{array}{l}\text { Working } \\
\text { memory } \\
\text { performance }\end{array}$ & Nicotine, Cytisine & $\begin{array}{l}\text { Acute administration } \\
\text { improved memory, while } \\
\text { chronic use reduced it }\end{array}$ \\
\hline Levin, 1996 & $\begin{array}{l}\text { Sprague } \\
\text { Dawley } \\
\text { rats }\end{array}$ & $\begin{array}{l}\text { Radial-arm } \\
\text { maze }\end{array}$ & $\begin{array}{c}\text { Working } \\
\text { memory } \\
\text { performance }\end{array}$ & Nicotine & $\begin{array}{l}\text { Nicotinic system improves } \\
\text { memory }\end{array}$ \\
\hline Levin, 1996 & $\begin{array}{l}\text { Sprague } \\
\text { Dawley } \\
\text { rats }\end{array}$ & $\begin{array}{l}\text { Radial-Arm } \\
\text { Maze }\end{array}$ & $\begin{array}{c}\text { Spatial working } \\
\text { memory }\end{array}$ & $\begin{array}{l}\text { Nicotine, Mecamylamine, } \\
\text { Scopolamine, Quinpirole. }\end{array}$ & $\begin{array}{c}\text { Acetylcholinesystems have } \\
\text { been shown to be important } \\
\text { for memory }\end{array}$ \\
\hline
\end{tabular}


FARMACIA, 2018, Vol. 66, 6

\begin{tabular}{|c|c|c|c|c|c|}
\hline Author, Year & Subjects & Tests utilised & $\begin{array}{l}\text { Evaluated } \\
\text { Parameter } \\
\end{array}$ & Chemical compounds & Comments \\
\hline Üzum, 2004 & Wistar rats & \begin{tabular}{|c|} 
Step-through \\
passive \\
avoidance task
\end{tabular} & $\begin{array}{l}\text { Learning and } \\
\text { memory } \\
\text { performance }\end{array}$ & Nicotine & $\begin{array}{l}\text { Acute and chronic nicotine } \\
\text { use enhances memory }\end{array}$ \\
\hline $\begin{array}{l}\text { Rojsanga, } \\
2012\end{array}$ & Mice & $\begin{array}{c}\text { Water maze } \\
\text { test }\end{array}$ & $\begin{array}{c}\text { Spatial learning } \\
\text { and memory }\end{array}$ & Crebanine & $\begin{array}{l}\text { Administration of crebanine } \\
\text { significantly improved the } \\
\text { cognitive deficits }\end{array}$ \\
\hline Hidaka, 2013 & Wistar rats & Y-maze test & $\begin{array}{l}\text { Short-term } \\
\text { working } \\
\text { memory }\end{array}$ & $\begin{array}{c}\text { ABT-418, physostigmine, } \\
\text { anabasine }\end{array}$ & $\begin{array}{c}\alpha 4 \beta 2 \text { agonist prevented } \\
\text { deficits in spontaneous } \\
\text { alternation behaviour }\end{array}$ \\
\hline $\begin{array}{l}\text { Arendash, } \\
1995\end{array}$ & $\begin{array}{l}\text { Sprague } \\
\text { Dawley } \\
\text { rats }\end{array}$ & \begin{tabular}{|c|} 
Lashley III \\
maze testing, \\
one-way active \\
avoidance \\
testing \\
\end{tabular} & $\begin{array}{c}\text { Memory } \\
\text { acquisition }\end{array}$ & GTS-21, DMAB & $\begin{array}{c}\text { The chronic administration } \\
\text { of nicotine enhances } \\
\text { cognitive functions }\end{array}$ \\
\hline $\begin{array}{l}\text { Arendash, } \\
1995\end{array}$ & $\begin{array}{l}\text { Sprague } \\
\text { Dawley } \\
\text { rats }\end{array}$ & $\begin{array}{c}\text { Lashley III } \\
\text { Maze, I7-Arm } \\
\text { radial maze, } \\
\text { one-way active } \\
\text { avoidance } \\
\end{array}$ & $\begin{array}{l}\text { Working } \\
\text { memory }\end{array}$ & Nicotine & $\begin{array}{l}\text { Nicotine pre-treatment } \\
\text { enhanced learning and } \\
\text { reference memory, but not } \\
\text { working memory }\end{array}$ \\
\hline $\begin{array}{l}\text { Nikiforuk, } \\
2015\end{array}$ & $\begin{array}{l}\text { Sprague } \\
\text { Dawley } \\
\text { rats }\end{array}$ & $\begin{array}{c}\text { Novel object } \\
\text { recognition, } \\
\text { attentional set- } \\
\text { shifting task, } \\
\text { Five-choice } \\
\text { serial reaction } \\
\text { time task }\end{array}$ & $\begin{array}{l}\text { Recognition } \\
\text { memory }\end{array}$ & $\begin{array}{l}\text { CCMI, PNU-120596, } \\
\text { galantamine, }\end{array}$ & $\begin{array}{l}\text { Nicotinic system improves } \\
\text { recognition memory }\end{array}$ \\
\hline $\begin{array}{l}\text { Camarasa, } \\
2010\end{array}$ & \begin{tabular}{c|} 
Long \\
Evans rats
\end{tabular} & \begin{tabular}{|c|} 
Object \\
recognition \\
test, Morris \\
water maze
\end{tabular} & $\begin{array}{c}\text { Spatial and non- } \\
\text { spatial learning } \\
\text { memory }\end{array}$ & Memantine (MEM) & $\begin{array}{l}\text { MEM prevents cognitive } \\
\text { deficits }\end{array}$ \\
\hline Kong, 2014 & Rats & $\begin{array}{c}\text { Open-field test } \\
\text { Morris water } \\
\text { maze test }\end{array}$ & $\begin{array}{c}\begin{array}{c}\text { Spatial learning } \\
\text { and memory }\end{array} \\
\end{array}$ & GTS-21 & $\begin{array}{l}\text { Nicotinic system improves } \\
\text { memory }\end{array}$ \\
\hline Hefco, 2003 & Wistar rats & \begin{tabular}{|c|} 
Passive \\
avoidance \\
task, Y-maze \\
\end{tabular} & $\begin{array}{l}\text { Long and short- } \\
\text { term memory }\end{array}$ & Scopolamine, Nicotine & $\begin{array}{l}\text { Nicotinic acetylcholine } \\
\text { receptors, improved short- } \\
\text { term memory }\end{array}$ \\
\hline Levin, 1997 & $\begin{array}{l}\text { Sprague } \\
\text { Dawley } \\
\text { rats }\end{array}$ & $\begin{array}{c}\text { Radial arm } \\
\text { maze, T-maze }\end{array}$ & $\begin{array}{l}\text { Working } \\
\text { memory }\end{array}$ & Nicotine, Mecamylamine. & $\begin{array}{l}\text { Nicotine induces receptor } \\
\text { binding, thus enhancing } \\
\text { memory }\end{array}$ \\
\hline
\end{tabular}

Table II

Studies using human subjects

\begin{tabular}{|c|c|c|c|}
\hline Author, Year & Study type & Subjects & Medication \\
\hline Lieberman, 2013 & Randomized Exploratory Trial & Patients with schizophrenia & TC-5619 \\
\hline Okada, 2013 & - & Patients with Alzheimer disease & F-2FA-85380 \\
\hline Lorenz, 2014 & - & Patients with idiopathic Parkinson's disease & - \\
\hline
\end{tabular}

Not only have the doses influenced the results, but the treatment duration as well. Studies have tried to make a comparison between acute and chronic administration of nicotine. In the case of acute nicotine administration studies, this compound had a significant positive effect on working memory. Moreover, its effect was reversed by administration of nicotinic antagonists [35]. In his study, Levin et al. concluded as well that chronic nicotine treatment determined an overall reduction in memory. On the other hand, Welzl et al. [61] determined that long- term nicotine treatment had little to no effect on cognition.

As above mentioned, the neuronal nicotinic receptors contribute to many cognitive functions, but not only. Most studies we encountered evaluate the effects of $\alpha 7$ stimulation, with the help of receptor agonists. Galantamine, PHAA-543613, GTS-21 and choline are only some of these chemical compounds studied over the years. Moreover, GTS-21, a selective $\alpha 7$ agonist, apart from its cognitive role, has been proven to have neuroprotective effects [29]. 
FARMACIA, 2018, Vol. 66, 6

Stimulating the dorsal hippocampal $\alpha 7$ nicotinic receptors, agonists could facilitate both memory consolidation, as well as reconsolidation shortly after accessing pieces of information [10]. Blake et al. [12] duplicated these results in a study performed later, when the authors highlighted as well the possibility to render new therapies for people recovering from amnesia.

When speaking about memory deficits developed under specific situations, it was assumed that the cholinergic system was involved as well. For example, in the case of hypoxia, a down-regulation of many receptors, including the $\alpha 7$-nicotinic receptor, has been observed [44]. This study concluded that the cognitive difficulties are determined by cholinergic dysfunctions.

Apart from this, it is well established that stress induces memory impairment. Alzoubi et al. [4] tried to determine if the nicotinic transmission had any effects in this particular condition. They used antagonists for different nicotinic receptor subtypes, which led to the conclusion that blocking the $\alpha 7$ receptors determined faster memory deterioration.

$\alpha 4 \beta 2$ nicotinic receptors have recently been connected with normal cognitive functions $[8,25,30]$. However, even though Kroker et al. reported the two subtypes having similar functions, they emphasized the fact that the $\alpha 7$ receptors had a higher efficacy than the $\alpha 4 \beta 2$ subtype. We also found publications that denied the $\alpha 4 \beta 2$ receptors having any effect on memory [4, 42].

Being localised not only in the hippocampus, but in the prefrontal cortex as well, the $\alpha 4 \beta 2$ were intimately linked to the working memory and attentional processes. Guillem et al. [21] determined this by observing an attention deficit in mice lacking the gene for the $\beta 2$ subunit. The same results were obtained by other studies as well [36, 51, 60].

Specific roles of the $\alpha 4 \beta 2$ nicotinic receptor have been studied. By using $\alpha 4 \beta 2$ partial agonists (e.g., ABT-1089), it resulted that this receptor subtype modulated memory impairment following nicotine withdrawal [63]. This finding might lead to new therapies for addiction. Even more, it is well known that in Parkinon's disease, not only the locomotion is affected but memory as well. The mechanisms of cognitive deficits have not been fully understood, but the cholinergic system has been connected to this condition, via the $\alpha 4 \beta 2$ nicotinic receptors, but not the $\alpha 7$ subtype [41].

As we have already mentioned, the entire cholinergic system is involved in memory development. Even though they have minimal effects, the muscarinic receptors participate as well in this cognitive process [28]. Liu et al. [40] determined that after blocking the nicotinic receptors, their function is taken by the muscarinic receptors. This study brought evidence that the muscarinic receptors are also involved in facilitating the spatial memory determined by nicotine. All types of nicotinic receptors are known to form complexes with one another in order to potentiate their functions. These receptors form complexes with a large number of other receptors, for example dopaminergic or serotoninergic. These complexes are proven to influence memory retrieval and consequently the working memory [24, 38, 54].

The most studied complexes that the nicotinic receptors form are the ones with the dopaminergic receptors. During different studies, it was observed that the cholinergic system stimulated the release of dopamine $[1,35]$. Acetylcholine interacts especially with the $\mathrm{D}_{2}$ receptors, thus potentiating the effect of nicotine on working memory. Using partial nicotinic agonists, such as varenicline, might have the same results on memory, but without leading to addiction [15]. Other authors have studied the participation of both $D_{1}$ and $D_{2}$ dopaminergic receptors. The administration of dopaminergic antagonists (for example cis-flupenthixol, which binds similarly to both $\mathrm{D}_{1}$ and $\mathrm{D}_{2}$ dopaminergic receptors) inhibits the stimulating effects that nicotinic agonists have on memory [13]. Kim et al. [28] determined that the interaction between nicotinic and dopaminergic systems influenced cognitive processes. The cholinergic-serotoninergic systems interaction has been extensively studied as well. Some studies concluded that overexpression of receptor complexes formed by the nicotinic receptors and $5-\mathrm{HT}_{1 \mathrm{~A}}$ receptor modulated memory deficits [58]. Moreover, Cristofor et al. [16] concluded that administration of paroxetine improved short-term spatial memory. Glutamate, through its receptors (AMPA and NMDA), has been strongly linked to the cholinergic system. A genetic abnormality in the AMPA receptors has been observed to determine a working memory impairment [54]. More importantly, the NMDA receptors have been connected to information acquisition $[14,54]$.

Apart from these, the $\mathrm{H}_{1}$ and $\mathrm{H}_{2}$ histamine receptors have been connected as well with cholinergic receptors. However, in this case, these two had conflicting effects. It was concluded that histamine inhibits the stimulating effect of the cholinergic system [18] on memory.

Either physiological (during the aging process) or pathological (precipitated by certain factors) neurodegeneration is an increasingly frequent issue in the general population. However, a well-established fact is that cognitive stimulation via the cholinergic system can prevent the degenerating process [8].

In the case of Alzheimer's disease (AD), several molecular markers have been detected. Blake et al. [11] observed that the density of $\alpha 4 \beta 2$ nicotinic receptors is decreased by $30-40 \%$. Okada et al. [47] studied the involvement of these receptor subtypes in non-smoking patients with Alzheimer's disease, coming to the same conclusion as previous studies. 
FARMACIA, 2018, Vol. 66, 6

However, the number of $\alpha 7$ receptors is not significantly modified. The same findings were observed in the case of cognitive deficits determined by chronic renal disease [7]. On the contrary, some studies concluded that $\alpha 7$ stimulation improved memory deficits existent in AD through a neurovascular mechanism [50, 53, 62]. The aetiology of the cognitive dysfunctions in schizophrenia has been studied in a number of clinical trials. For example, Lierberman et al. [39] observed the positive effects certain $\alpha 7$ agonists (as an example, TC-5619) had in this situation. Speaking about Parkinson's disease, there were similar findings. Samnick et al. [41] used imaging techniques to determine the role of nicotinic receptors in memory impairment. Their results were consistent with previous studies and showed the involvement of nicotinic receptors in the cognitive processes in Parkinson's disease.

Following all knowledge, treatments with nicotinic agonists have been considered for improving cognitive deficits in neurodegenerative diseases, but not only $[53,62]$. However, treatments for such complex diseases might necessitate the administration of multiple substances. That is why further studies need to be performed and clinical trials should establish treatment protocols.

\section{Conclusions}

Memory formation is a complex process, with a large number of steps, each mediated via neurotransmitter systems. The most important contribution is brought by cholinergic receptors, which enhance both information acquisition and memory consolidation. They frequently form receptor complexes with the dopaminergic, serotoninergic, histaminergic and NMDA receptors. In conclusion, memory in pathologic conditions such as neurodegenerative diseases, stress induced cognitive deficits, chronic renal disease and nicotine withdrawal can be improved by the cholinergic transmission via nicotinic receptors alongside other receptors such as the dopaminergic, the serotoninergic and NMDA receptors.

\section{References}

1. Addy N, Levin ED, Nicotine Interactions with Haloperidol, Clozapine and Risperidone and Working Memory Function in Rats. Neuropsychopharmacology, 2002; 27(4): 534-541.

2. Addy NA, Nakijama A, Levin ED, Nicotinic mechanisms of memory: effects of acute local DHbE and MLA infusions in the basolateral amygdala. Cogn Brain Res., 2003; 16: 51-57.

3. Alkondon M, Pereira EF, Albuquerque EX, Alpha bungarotoxin- and methyllcaconitine-sensitive nicotinic receptors mediate fast synaptic transmission in interneurons of rat hippocampal slices. Brain Res., 1998; 810(1-2): 257-263.
4. Alzoubi KH, Srivareerat M, Tran TT, Alkadhi KA, Role of a7- and a4b2-nAChRs in the neuroprotective effect of nicotine in stress-induced impairment of hippocampus-dependent memory. Int $J$ Neuropsychopharmacology, 2013; 16: 1105-1113.

5. Arendash GW, Sengstock GJ, Sanberg PR, Kem WR, Improved learning and memory in aged rats with chronic administration of the nicotinic receptor agonist GTS-21. Brain Res., 1995; 674: 252-259.

6. Arendash GW, Sanberg PR, Sengstock GJ, Nicotine Enhances the Learning and Memory of Aged Rats. Pharmaco Biochem Behav., 1995; 52(3): 517-523.

7. Ballesta JJ, Pozo C, Castelló-Banyuls J, Faura CC, Selective down-regulation of $\alpha 4 \beta 2$ neuronal nicotinic acetylcholine receptors in the brain of uremic rats with cognitive impairment. Exp Neurol., 2012; 236: 28-33.

8. Baraldi T, Schöwe Mendes N, Balthazar J, MonteiroSilva KC, Albuquerque MS, Buck HS, Viel TA, Cognitive stimulation during lifetime and in the aged phase improved spatial memory, and altered neuroplasticity and cholinergic markers of mice. Exp Gerontol., 2013; 48: 831-838.

9. Bettany JH, Levin ED, Ventral hippocampal a7 nicotinic receptor blockade and chronic nicotine effects on memory performance in the radial-arm maze. Pharmacol Biochem Behav., 2001; 70: 467-474.

10. Blake MG, Boccia MM, Krawczyk MC, Delorenzi A, Choline reverses scopolamine-induced memory impairment by improving memory reconsolidation. Neurobiol Learn Memory, 2012; 98: 112-121.

11. Blake MG, Boccia MM, Krawczyk MC, Baratti CM, Hippocampal a7-nicotinic cholinergic receptors modulate memory reconsolidation: A potential strategy for recovery from amnesia. Neurobiol Learn Memory, 2013; 106: 193-203.

12. Blake MG, Krawczyk MC, Baratti CM, Boccia MM, Neuropharmacology of memory consolidation and reconsolidation: Insights on central cholinergic mechanisms. J Physiol., 2014; 108(4-6): 286-291.

13. Brioni JD, Arneric SP, Nicotinic Receptor Agonists Facilitate Retention of Avoidance Training: Participation of Dopaminergic Mechanisms. Behav Neural Biol., 1993; 59: 57-62.

14. Camarasa J, Rodrigo T, Pubill D, Escubedo E, Memantine is a useful drug to prevent the spatial and non-spatial memory deficits induced by methamphetamine in rats. Pharmacol Res., 2010; 62: 450-456.

15. Chirila M, Ghita I, Fulga I, Current knowledge on brupropion and varenicline clinical efficacy in nicotine dependence. Farmacia, 2015; 63(1): 1-7.

16. Cristofor AC, Mititelu Tartau L, Popa G, Lupusoru RV, Chirita V, Bibire N, Rusu G, Lupusoru CE, The influence of some antidepressant drugs on memory in rats. Farmacia, 2015; 63(4): 526-529.

17. Deutsch SI, Burket JA, Benson AD, Targeting the $\alpha 7$ nicotinic acetylcholine receptor to prevent progressive dementia and improve cognition in adults with Down's syndrome. Prog Neuro-Psychopharmacolology Biol Psych., 2014; 54: 131-139.

18. Eidi M, Mohammad-Reza Z, Eidi A, Oryan S, Parivar $\mathrm{K}$, Effects of histamine and cholinergic systems on memory retention of passive avoidance learning in rats. Eur J Pharmacol., 2003; 465: 91-96. 
FARMACIA, 2018, Vol. 66, 6

19. Falsafi SK, Deli A, Hoger H, Pollak A, Lubec G, Scopolamine Administration Modulates Muscarinic, Nicotinic and NMDA Receptor Systems. PloS ONE, 2012; 7(2): e32082: 1-12.

20. Flood JF, Landry DW, Jarvik M, Cholinergic receptor interactions and their effects on long-term processing. Brain Res., 1981; 215: 177-185.

21. Guillem K, Bloem B, Poorthuis RB, Loos M, Smit AB, Maskos U, Spijker S, Mansvelder HD, Nicotinic acetylcholine receptor beta2 subunits in the medial prefrontal cortex control attention. Science, 2011; 333: 888-891.

22. Guo LL, Guan ZZ, Wang YL, Scutellarin protects against $A \beta$-induced learning and memory deficits in rats: involvement of nicotinic acetylcholine receptors and cholinesterase. Acta Pharmacol Sinica, 2011; 32: 1446-1453.

23. Haroutounian V, Barnes E, Kenneth LD, Cholinergic modulation of memory in rats. Psychopharmacology, 1985; 87: 266-271

24. Hefco V, Yamada K, Hefco A, Hritcu L, Tiron A, Olariu A, Nabeshima T, Effects of nicotine on memory impairment induced by blockade of muscarinic, nicotinic and dopamine D2 receptors in rats. Eur $J$ Pharmacol., 2003; 474: 227- 232.

25. Hidaka N, Suemaru K, Kato Y, Araki H, Involvement of $\mathrm{a} 4 \mathrm{~b} 2$ nicotinic acetylcholine receptors in working memory impairment induced by repeated electroconvulsive seizures in rats. Epilepsy Res., 2013; 104: 181-185.

26. De Jonge WJ, Ulloa L, The alpha7 nicotinic acetylcholine receptor as a pharmacological target for inflammation. Br J Pharmacol., 2007; 151(7): 915-929.

27. Karamihalev S, Prickaerts J, van Goethem NP, Donepezil and the alpha-7 agonist PHA 568487, but not risperidone, ameliorate spatial memory deficits in a subchronic MK-801 mouse model of cognitive impairment in schizophrenia. Behav Brain Res., 2014; 272: 248-251.

28. Kim JS, Levin ED, Nicotinic, muscarinic and dopaminergic actions in the ventral hippocampus and the nucleus accumbens: effects on spatial working memory in rats. Brain Res., 1996; 725: 231-240.

29. Kong FJ, Ma LL, Zhang HH, Zhou JQ, Alpha 7 nicotinic acetylcholine receptor agonist GTS-21 mitigates isoflurane-induced cognitive impairment in aged rats. J Surg Res., 2015; 194(1): 255-261.

30. Kroker KS, Georg R, Holger R, Differential effects of subtype-specific nicotinic acetylcholine receptor agonists on early and late hippocampal LTP. Eur $J$ Pharmacol., 2011; 671: 26-32.

31. Lange-Asschenfeldt C, Schable S, Suvorava T, Fahimi Ehsan G, Bisha M, Stermann T, Henning U, Effects of varenicline on alpha4-containing nicotinic acetylcholine receptor expression and cognitive performance in mice. Neuropharmacology, 2016; 107: 100-110.

32. Leiser SC, Bowlby MR, Comery TA, Dunlop J, A $\operatorname{cog}$ in cognition: How the $\alpha 7$ nicotinic acetylcholine receptor is geared towards improving cognitive deficits. Pharmacol Therap., 2009; 122: 302-311.

33. Levin ED, Briggs SJ, Christopher NC, Rose JE, Persistence of Chronic Nicotine-Induced Cognitive Facilitation. Behav Neural Biol., 1992; 58: 152-158.
34. Levin ED, Briggs SJ, Christopher NC, Rose JE, Prenatal Nicotine Exposure and Cognitive Performance in Rats. Neurotox Terat., 1993; 15: 251-260.

35. Levin ED, Rose JE, Acute and Chronic Nicotinic Interactions with Dopamine Systems and Working Memory Performance. Ann NY Acad Sci., 1995; 757: 245-252.

36. Levin ED, Torry D, Acute and chronic nicotine effects on working memory in aged rats. Psychopharmacology, 1996; 123: 88-97.

37. Levin ED, Kim P, Meray R, Chronic nicotine working and reference memory effects in the 16-arm radial maze: interactions with D1 agonist and antagonist drugs. Psychopharmacology, 1996; 127: 25-30.

38. Levin ED, Torry D, Christopher NC, Yu X, Einstein G, Schwartz-Bloom RD, Is Binding to Nicotinic Acetylcholine and Dopamine Receptors Related to Working Memory in Rats?. Brain Res Bull., 1997; 43(3): 295-304.

39. Lieberman JA, Dunbar G, Segreti AC, Girgis RR, Seoane F, Beaver JS, Duan N, Hosford DA, A randomized exploratory trial of an alpha-7 nicotinic receptor agonist (TC-5619) for cognitive enhancement in schizophrenia. Neuropsychopharmacology, 2013; 38: 968-975.

40. Liu Y, Wang Y, Su X, Evidence that muscarinic receptors are involved in nicotine-facilitated spatial memory. Pharmacol Biochem Behav., 2004; 78: 775-779.

41. Lorenz R, Samnick S, Dillmann U, Schiller M, Ong MF, Fabender K, Buck A, Spiegel J, Nicotinic a4b2 acetylcholine receptors and cognitive function in Parkinson's disease. ActaNeurolScand., 2014; 130: 164-171.

42. Morris KA, Li S, Bui DD, Gold PE, Glucose attenuates impairments in memory and CREB activation produced by an a $4 \mathrm{~b} 2$ but not an a 7 nicotinic receptor antagonist. Neuropharmacology, 2013; 67: 233-242.

43. Mundy WR, Iwamoto ET, Nicotine Impairs Acquisition of Radial Maze Performance in Rats. Pharmacol Biochem Behav., 1988; 30: 119-122.

44. Muthuraju S, Panchanan M, Soumya P, Preeti S, Alpesh KS, Shashi BS, Dipti P, Govindasamy I, Role of cholinergic markers on memory function of rats exposed to hypobaric hypoxia. Eur J Pharmacol., 2011; 672: 96-105.

45. Nader K, Shafe GE, Le Doux JE, Fear memories require protein synthesis in the amygdale for reconsolidation after retrieval. Nature, 2000; 406(6797): $722-726$.

46. Nikiforuk A, Kos T, Potasiewicz A, Popik P, Positive allosteric modulation of alpha 7 nicotinic acetylcholine receptors enhances recognition memory and cognitive flexibility in rats. Eur Neuropsychopharmacol., 2015; 25(8): 1300-1313.

47. Okada H, Ouchi Y, Ogawa M, Futatsubashi M, Saito Y, Yoshikawa E, Terada T, Oboshi Y, Tsukada H, Ueki T, Watanabe M, Yamashita T, Alterations in a4b2 nicotinic receptors in cognitive decline in Alzheimer's etiopathology. Brain, 2013; 136: 30043017.

48. Perio A, Terranova JP, Worms P, Bluthe RM, Dantzer R, Biziere K, Specific modulation of social memory in rats by cholinomimetic and nootropic drugs, by 
benzodiazepine inverse agonists, but not by psychostimulants. Psychopharmacology, 1989; 97: 262-268.

49. Puma C, Deschaux O, Molimard R, Bizot JC, Nicotine improves memory in an object recognition task in rats. Eur Neuropsychopharmacol., 1999; 9: 323-327.

50. Rojsanga $\mathrm{P}$, Boonyarat $\mathrm{C}$, Utsintong $\mathrm{M}$, Nemecz Á, Yamauchi JG, Talley TT, Olson AJ, Matsumoto K, Vajragupta $\mathrm{O}$, The effect of crebanine on memory and cognition impairment via the alpha-7 nicotinic acetylcholine receptor. Life Sci., 2012, 91, 107-114.

51. Rushforth SL, Allison C, Wonnacott S, Shoaib M, Subtype-selective nicotinic agonists enhance olfactory working memory in normal rats: A novel use of the odour span task. Neurosci Lett., 2010; 471: 114-118.

52. Stoyo Karamihalev, Jos Prickaerts, Donepezil and the alpha-7 agonist PHA 568487, but not risperidone, ameliorate spatial memory deficits in a subchronic MK-801 mouse model of cognitive impairment in schizophrenia. Behav Brain Res., 2014; 272: 248-251.

53. Sadigh-Eteghad S, Talebi M, Mahmoudi J, Babri S, Shanehbandi D., Selective activation of a7 nicotinic acetylcholine receptor by PHA-543613 improves Ab25-35-mediated cognitive deficits in mice. Neuroscience, 2015; 298: 81-93.

54. Shanmugasundaram B, Sase A, Miklosi AG, Sialana FJ, Subramaniyan S, Aher YD, Gröger M, Frontal cortex and hippocampus neurotransmitter receptor complex level parallels spatial memory performance in the radial arm maze. Behav Brain Res., 2015; 289: $157-168$.

55. Smith RD, Kistler MK, Cohen-Williams M, Coffin VL, Cholinergic improvement of a naturally-occurring memory deficit in the young rat. Brain Res., 1996; 707: 13-21.

56. Socci DJ, Sanbergi PR, Arendash GW, Nicotine Enhances Morris Water Maze Performance of Young and Aged Rats. Neurobiology of Aging, 1995; 16(5): 857-860.
57. Stolerman IP, Mirza NR, Hahn B, Shoaib M, Nicotine in an animal model of attention. Eur J Pharmacol., 2000; 393: 147-154.

58. Subramaniyan S, Heo S, Patil SS, Li L, Hoger H, Pollak A, A hippocampal nicotinic acetylcholine alpha 7-containing receptor complex is linked to memory retrieval in the multiple-T-maze in C57BL/6j mice. Behav Brain Res., 2014; 270: 137-145.

59. Uzum G, Diler AS, Nesrin B, Mustafa T, Yurekli Y, Ziylan Z, Nicotine improves learning and memory in rats: Morphological evidence for acetylcholine involvement. Intern J Neurosci., 2004; 114: 1163-1179.

60. Vandesquille M, Baudonna M, Decorte L, Louis C, Pierre L, Béracochéa D, Working memory deficits and related disinhibition of the cAMP/PKA/CREB are alleviated by prefrontal a4b2-nAChRs stimulation in aged mice. Neurobiology of Aging, 2013; 34: 1599-1609.

61. Welzl H, Alessandri B, Oettinger R, Battig K, The effects of long-term nicotine treatment on locomotion, exploration and memory in young and old rats. Psychopharmacology, 1988; 96: 317-323.

62. Werkheiser JL, Sydsreff S, Hubbs SJ, Ding M, Eisman MS, Perry D, Williams AJ, Smith JS, Mrzljak L, Ultra-low exposure to alpha-7 nicotinic acetylcholine receptor partial agonists elicits an improvement in cognition that corresponds with an increase in alpha-7 receptor expression in rodents: implications for low dose clinical efficacy. Neuroscience, 2011; 186: 76-87.

63. Yildirim E, Connor DA, Gould TJ, ABT-089, but not ABT-107, ameliorates nicotine withdrawal-induced cognitive deficits in C57BL6/J mice. Behav Pharmacol., 2015; 26(3): 241-248.

64. Zhou M, Suszkiw JB, Nicotine attenuates spatial learning deficits induced in the rat by perinatal lead exposure. Brain Res., 2004; 999: 142-147. 